\title{
Assessment of Sentinel-1A/B SAR Derived Ocean Wind Speeds against HY-2B Scatterometer in the Presence of Ocean Swells
}

\author{
He Wang ${ }^{1{ }^{*}}$, Chaoying $\mathrm{Shi}^{2}$ and Jianhua $\mathrm{Zhu}^{1}$ \\ ${ }^{1}$ National Ocean Technology Center, Ministry of Natural Resources, Tianjin, 300112, China \\ ${ }^{2}$ National Center of Ocean Standards and Metrology, Ministry of Natural Resources, Tianjin, 300112 , \\ China
}

\begin{abstract}
Sentinel-1A/B satellites operating in wave mode provide ocean winds dataset on a continuous and global basis. In this study, wind speeds derived from Sentinel-1A/B wave mode imagery from November 2018 to October 2020 are evaluated against HSCAT scatterometer aboard Chinese satellite HY-2B. Here, due to the close equatorial crossing times between Sentinel-1 and HY-2B, the spatio-temporal criteria of $50 \mathrm{~km}$ and $30 \mathrm{~min}$ yield large amount match-ups. Comparison results show a good agreement between wind speeds derived from the two types of radars: synthetic aperture radar and scatterometer. Impact of the presence of pure swell on the evaluation results is also discussed.
\end{abstract}

\section{Introduction}

Observations of ocean winds are important for various scientific and engineering areas. For decades, the ocean winds are provided from space by remote sensor of scatterometers and synthetic aperture radar (SAR) instruments [1][2]. SAR offers unique spaceborne ocean wind data due to its wide coverage and fine resolution. The recent C-band SAR satellites are Sentinel-1A/B, which launched by European Space Agency (ESA) in 2014 and 2016 in the framework of Copernicus program. Nowadays, Sentinel-1A/B SAR constellation is routinely monitoring the global oceans in the special SAR configuration of wave mode. However, the detailed quality assessment on the Sentinel-1A/B SAR ocean wind products is of importance for user community of ocean and atmosphere.

As active radars, SARs measure the modulation of normalized radar cross section (NRCS) from the wind roughened sea surface, which a function of sea surface winds in the first order. Therefore, ocean surface winds can be retrieved from SAR based on the geophysical model functions (GMF) relating NRCS and winds for specific radar configuration [3]. However, it is evident that ocean swell is a factor impacting wind speed retrieval from $\mathrm{C}$-band SARs. The main mechanism for this is that the large-scale waves (e.g., the long-period swells) impacts the small-scale roughness deduced by winds, consequently, changes the radar backscattering over the ocean [4][5].

\footnotetext{
*Corresponding author: wanghe_sio@126.com
} 
In this context, although some efforts have been devoted for the performance assessment of winds derived from Sentinel-1 SAR imagery (e.g., [6][7]), the SAR winds quality assessment under complex non-wind background, for instance swells, is still needed detailed studies. To better address and understand the quality of Sentinel-1A/B winds, we aim at comprehensively quantifying the performance of SAR derived winds by collocating HY-2B scatterometer, especially for the presence of ocean swells.

\section{Data sets}

\subsection{Sentinel-1A/B wave mode data}

Sentinel-1A/B wave mode operates in the so-called "leap frog" acquisition mode, in which SAR image is acquired every $100 \mathrm{~km}$ at two alternating incidence angles $\left(23^{\circ}\right.$ and $36^{\circ}$, and called WV1 and WV2, respectively), with two images acquired at the same incidence 200 $\mathrm{km}$ apart in the open ocean. Each Sentinel-1 SAR image, is about $20 \mathrm{~km}$ swath with spatial resolution around $5 \mathrm{~m}$ in single polarization of VV as default.

In Ocean Wind Fields (OWI) of Level-2 products, the ocean wind speeds are derived from the calibrated SAR NRCS (Level-1) using Geophysical model function (GMF) of CMOD-IFR2 with ECMWF wind direction as a priori input. In addition, the classification of Sentinel-1A/B wave mode SAR scenes has been automatically produced and routinely released by Institut Français de Recherche pour l'Exploitation de la Mer (IFREMER), based on deep learning convolutional neural network tool [7]. Here, only the geophysical phenomena labelled as ocean swell and wind streaks are remained. The typical Sentinel-1 SAR scenes classified as wind streaks and ocean swell are shown in figure 1.
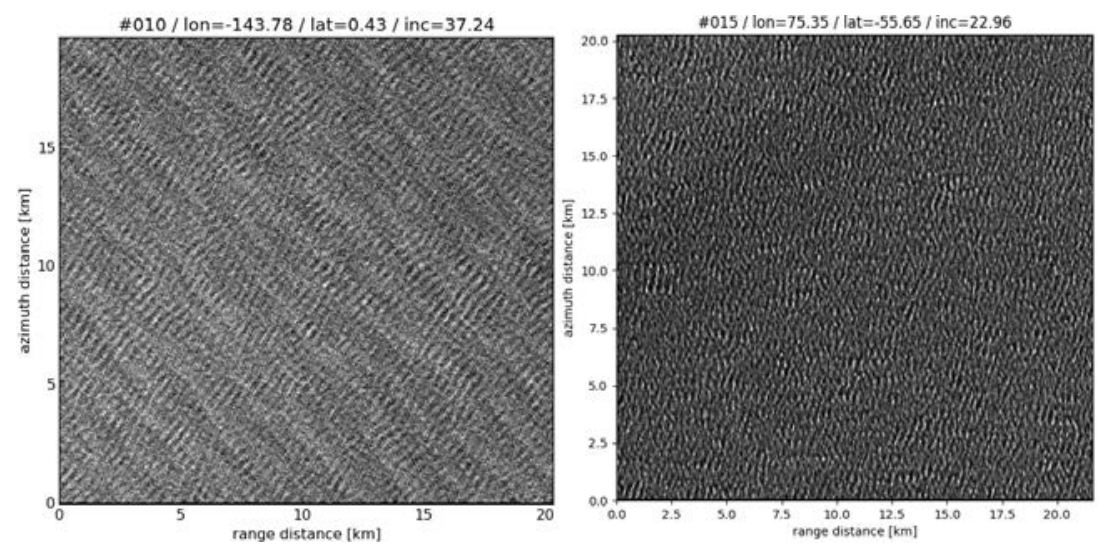

Fig. 1. Typical (left) wind streaks and (right) pure ocean swells Sentinel-1 SAR imagery.

\subsection{HY-2B scatterometer winds}

HSCAT aboard Chinese satellite HY-2B launched in 2018 is a conically scanning scatterometer operating in $\mathrm{Ku}$-band $(13.256 \mathrm{GHz})$. In swaths of $1350 \mathrm{~km}$ and $1750 \mathrm{~km}$, its two beams are alternately transmitting vertically and horizontally polarized Ku-band microwave pulses, respectively. Officially, two types of retrieval algorithms produce level 2B winds from HY-2B HSCAT, namely PWP and DPS. Here, we used the level 2B winds derived using PWP algorithm, which is based on the prototype developed in Royal 
Netherlands Meteorological Institute (KNMI), since the better performance than those from DPS [8].

\section{Results}

\subsection{Co-location of Sentinel-1A/B and HY-2B}

The match-up dataset was built by col-locating the Sentinel-1A/B and HY-2B HSCAT within the spatio-temporal criteria of $25 \mathrm{~km}$ and $30 \mathrm{~min}$, respectively. This threshold yields approximately 0.5 million globally distributed data pairs as shown in figure 2 for wind streaks and ocean swell SAR scenes, respectively.
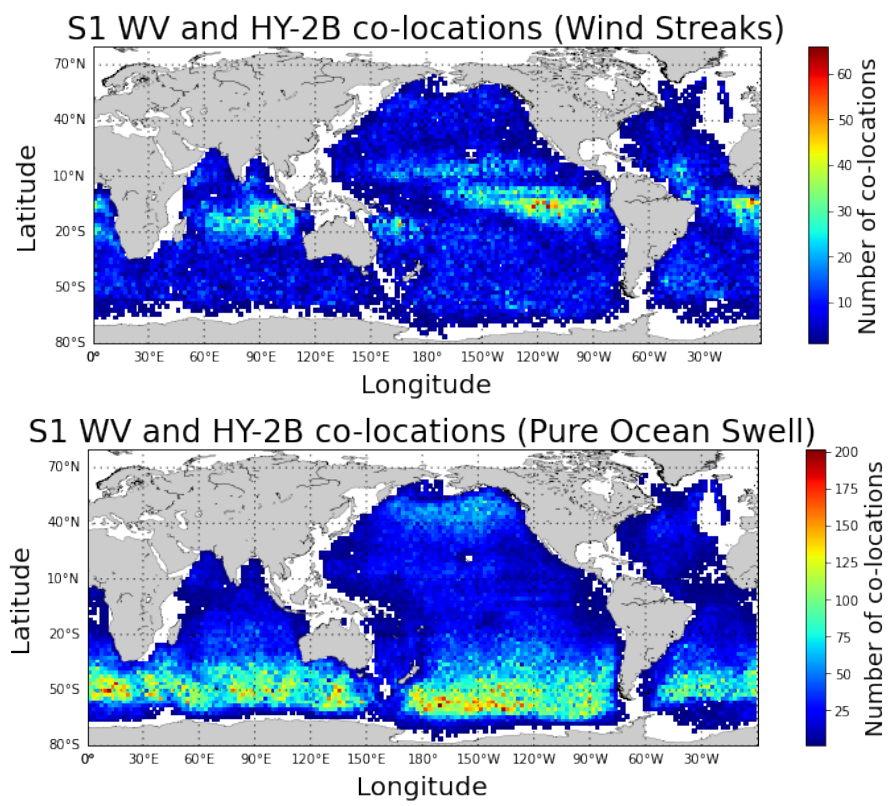

Fig. 2. Geographical distributions of Sentinel-1A/B and HY-2B co-locations for wind streaks and ocean swell SAR scenes.

\subsection{Assessment metrics}

The statistics of bias, Root Mean Square Error (RMSE), the Scatter Index (SI), and correlation coefficient (COR) used in the evaluation are expressed by:

$$
\begin{aligned}
& \text { bias }=\frac{1}{N} \sum_{i=1}^{N}\left(x_{i}-y_{i}\right) \\
& \text { RMSE }=\sqrt{\frac{1}{N} \sum_{j=1}^{N}\left(x_{i}-y_{i}\right)^{2}}
\end{aligned}
$$




$$
\begin{aligned}
& \mathrm{SI}=\frac{1}{\left\langle\mathrm{x}_{\mathrm{i}}\right\rangle} \sqrt{\frac{1}{\mathrm{~N}} \sum_{\mathrm{i}=1}^{\mathrm{N}}\left[\left(\mathrm{y}_{\mathrm{i}}-\left\langle\mathrm{y}_{\mathrm{i}}\right\rangle\right)-\left(\mathrm{x}_{\mathrm{i}}-\left\langle\mathrm{x}_{\mathrm{i}}\right\rangle\right)\right]^{2}} \times 100 \% \\
& \mathrm{COR}=\frac{\sum_{\mathrm{i}=1}^{\mathrm{N}}\left(\mathrm{x}_{\mathrm{i}}-\left\langle\mathrm{x}_{\mathrm{i}}\right\rangle\right)-\left(\mathrm{y}_{\mathrm{i}}-\left\langle\mathrm{y}_{\mathrm{i}}\right\rangle\right)}{\sqrt{\sum_{\mathrm{i}=1}^{\mathrm{N}}\left(\mathrm{x}_{\mathrm{i}}-\left\langle\mathrm{x}_{\mathrm{i}}\right\rangle\right)^{2} \sum_{\mathrm{i}=1}^{\mathrm{N}}\left(\mathrm{y}_{\mathrm{i}}-\left\langle\mathrm{y}_{\mathrm{i}}\right\rangle\right)^{2}}}
\end{aligned}
$$

where xi and yi represent the remotely sensed wind speed from Sentinel-1A/B wave mode SAR and HY-2B scatterometer, and $\mathrm{N}$ is the sample size.

\subsection{Validation results over wind/swell scenarios}

Figure 3 shows the comparison result of Sentinel-1A/B wind speed retrievals against HY2B HSCAT data for wind streaks scenarios. Based upon the matchups, we also investigate the influence of SAR radar incidence angle on the validation results. SAR wind estimation performance of about $1.0 \mathrm{~m} / \mathrm{s}$ RMSE, $\sim 10.9 \%$ SI and negligible bias is presented for both incident angles. Moreover, from the overlaid error bars representing the standard deviation for each wind speed bin, one could see underestimation and the relatively large RMSEs at high wind speeds, and the degraded quality in low wind condition.


Fig. 3. Scatter plots of Sentinel-1A/B wind speeds on wind streaks scenes versus HY-2B products for SAR incidence angle of $23^{\circ}$ (left) and $36^{\circ}$ (right), respectively.

In the terms of pure swells scenarios, figure 4 shows the validation results for two SAR radar incidence angles. Larger RMSEs $\left(1.35 \mathrm{~m} / \mathrm{s}\right.$ and $1.47 \mathrm{~m} / \mathrm{s}$ for $23^{\circ}$ and $36^{\circ}$, respectively), and SI $(\sim 14 \%) \%$ could be found. It is indicated that swells have remarkable influence on Sentinel-1 SAR wind retrievals. 


\section{EDEP 2021}

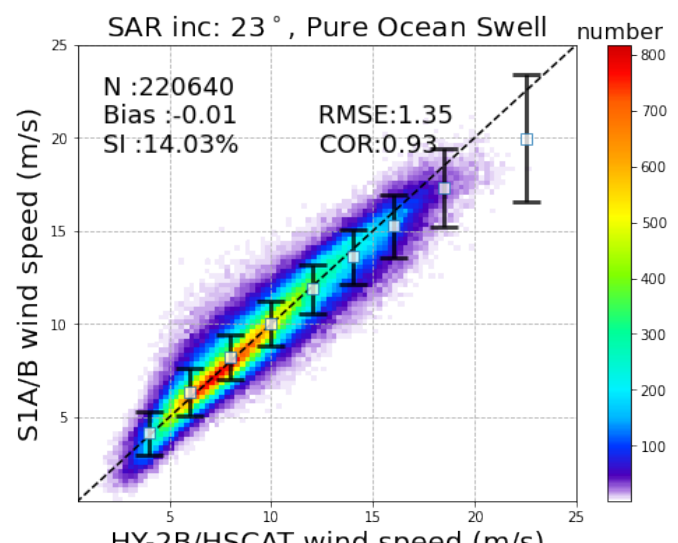

HY-2B/HSCAT wind speed $(\mathrm{m} / \mathrm{s})$

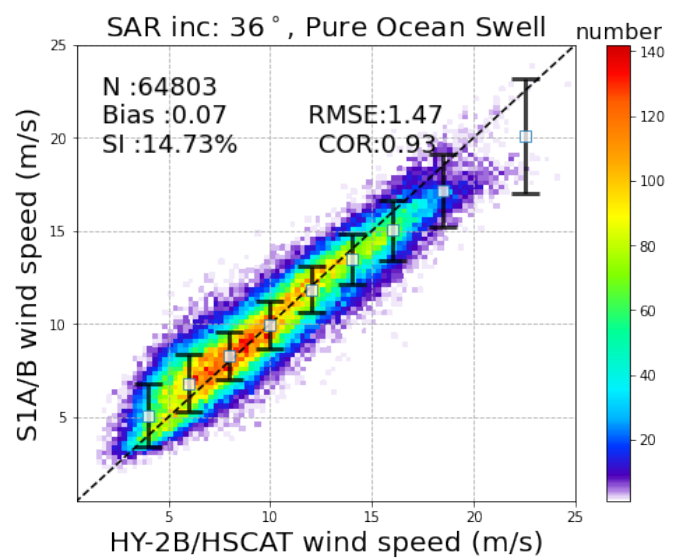

Fig. 4. As in figure 3, but for in the presence of pure swells.

\section{Conclusion}

In this paper, Sentinel-1A/B imagery and HY-2B HSCAT were co-located within spatiotemporal criteria of $50 \mathrm{~km}$ and $30 \mathrm{~min}$, resulting to a large dataset for both swell and wind scenes, due to the close equatorial crossing times between each other.

Based on co-locations, wind speeds derived from sentinel-1A/B wave mode were validated against HSCAT scatterometer for the period of two years. Comparison results show a good agreement between from Sentinel-1A/B and HY-2B with respect to the wind speed for pure wind scenarios. The assessment also shows the considerable influence of swells co-existence on the sentinel-1A/B wind performance. Impact of radar incidence angle of Sentinel-1A/B on the comparison results is also investigated.

In order to minimized the swell impact, one possible treatment is to adding swell heights information when retrieving ocean surface winds. This would upgrade the present operational retrieval schemes respective to the ocean swell influence. 


\section{References}

[1] Stoffelen A C and Anderson D L 1997 Scatterometer data interpretation: Measurement and inversion J. Atmos. Ocean. Technol. 14 1298-1313

[2] Wang H, Yang J, Mouche A, Shao W, Zhu J, Ren L and Xie C 2017 GF-3 SAR Ocean Wind Retrieval: The First View and Preliminary Assessment Remote Sens. 9694

[3] Stoffelen A, Verspeek J A, Vogelzang J and Verhoef A 2017 The CMOD7 Geophysical Model Function for ASCAT and ERS Wind Retrievals. IEEE J. Selected Top. Appl. Earth Observ. Remote Sens. 10 2123-2134

[4] Wang H, Yang J, Zhu J, Ren L, Liu Y, Li W and Chen C 2021 Estimation of Significant Wave Heights from ASCAT Scatterometer Data via Deep Learning Network Remote Sens. 2021, 13195

[5] Li H, Mouche A, and Stopa J E 2019 Impact of Sea State on Wind Retrieval From Sentinel-1 Wave Mode Data IEEE J. Selected Top. Appl. Earth Observ. Remote Sens. 12 559-566

[6] Monaldo F, Jackson C, Li X and Pichel W G 2016 Preliminary Evaluation of Sentinel1A Wind Speed Retrievals IEEE J. Selected Top. Appl. Earth Observ. Remote Sens. 9 2638-2642

[7] Bentamy A, Mouche A, Grouazel A, Moujane A and Mohamed A A 2018 Using sentinel-1A SAR wind retrievals for enhancing scatterometer and radiometer regional wind analyses Int. J. Remote Sens. 40 1120-1147

[8] Wang C, Tandeo P, Mouche A, Stopa J E, Gressani V, Longepe N, Vandemark D, Foster R C and Chapron B 2019 Classification of the global Sentinel-1 SAR vignettes for ocean surface process studies Remote Sens. Environ. 234111457

[9] Wang H, Zhu J, Lin M, Zhang Y and Chang Y 2020 Evaluating Chinese HY-2B HSCAT Ocean Wind Products Using Buoys and Other Scatterometers IEEE Geosci. Remote Sens. Lett. 17 923-927 